UDK 781:001.8

Dalibor Davidović

Akademija za glasbo Univerze $\mathrm{v}$ Zagrebu

Music Academy, University of Zagreb

\title{
Beispiele der Theoriebildung in der Musikwissenschaft
}

\section{Einführende Bemerkungen}

Auf dem ersten Blick kann der Titel recht unbestimmt scheinen, und die Erwartungen, die er erweckt, werden wohl sehr unterschiedlich sein. Man könnte sie einigermaßen konkretisieren, indem einige Anregungen zum Thema gegeben werden. Da es jetzt natürlich nicht möglich ist, alle Anregungen zu erwähnen, werde ich mich nur auf diejenigen konzentrieren, die mir für das Verständnis dieses Aufsatzes wichtig erscheinen. Ich werde sie einfach als eine Art Fragenkatalog darstellen, wobei ihre eigenen Voraussetzungen in diesem Moment nicht diskutiert werden.

\section{Anregungen}

Eine der wichtigsten Fragen für diese Arbeit kann man aus dem Text "Unverständliche Wissenschaft: Probleme einer theorieeigenen Sprache" von Niklas Luhmann (Luhmann 1981) entnehmen. Dort behandelt Luhmann das Problem der Anknüpfung an die terminologische Tradition einer wissenschaftlichen Disziplin. Laut Luhmann sind in einem solchen Fall zwei Optionen möglich: "Terminologien zu kontinuieren, obwohl ihre Bedeutung sich ändert, oder sie aufzugeben, und damit auf Identifikationslinien zur Tradition hin zu verzichten." (173) Falls diese zweite Option jedoch das Greifen nach einer im Rahmen einer anderen Disziplin geschaffenen Terminologie darstellt, entsteht nach Luhmann einerseits auch das Problem der Kontrolle, die eine Disziplin dadurch bezüglich einer anderen übernimmt, während andererseits das Problem einer gewissen Inflationierung des theoretischen Jargons der Disziplin erscheint, aus der das Vokabular entliehen wurde (175). Es ist bekannt, dass diese zweite Option, von der Luhmann schreibt, in der Wissenschaft sehr weit verbreitet ist, nicht nur in der letzten Zeit. Es scheint aber, dass sie in der letzten Zeit besonders häufig als wünschenswert dargestellt 
wird, und Musikwissenschaft hier keineswegs eine Ausnahme ist. Die Option, um die es sich hier handelt, ist nämlich der wichtigste Schritt innerhalb jenes wissenschaftlichen Programms, das unter dem Namen Interdisziplinarität bekannt ist. Luhmanns Beitrag zur Diskussion scheint insofern wichtig, da aus ihm zu entnehmen ist, dass die Interdisziplinarität als Programm doch nicht so unproblematisch ist, wie es auf dem ersten Blick scheinen mag, sondern, dass man bei einem Theorietransfer in eine andere Disziplin zumindest mit gewissen Nebenwirkungen rechnen sollte.

Gerade die Frage, wie dieser Transfer konkret läuft und welche Nebenwirkungen er hat, ist in der neueren Zeit zu einem wichtigen Thema in einigen Disziplinen geworden. Wie die eigene Disziplin eine andere beobachtet hat, ist besonders in der neueren Literaturwissenschaft eine häufig gestellte Frage. Es sind hier nur zwei Beispiele zu erwähnen: Das erste ist die Diskussion, die größtenteils in den Achtzigern geführt wurde, und in derem Zentrum der Transfer der Dekonstruktion Jacques Derridas in die US-amerikanische Literaturwissenschaft lag (ein Beispiel ist Norris 1988; eine Beobachtung der amerikanischen Diskussion aus der Position der Literaturwissenschaft in Deutschland ist in Gumbrecht $1988 \mathrm{zu}$ finden); das zweite Beispiel aus der Literaturwissenschaft ist die neuere Diskussion um den literaturwissenschaftlichen Import der soziologischen Systemtheorie, wie sie durch Luhmann vertreten wird. Die niederländischen Theoretiker Tannelie Blom und Ton Nijhuis (Blom/Nijhuis 1995) haben das Problem des Theorietransfers in diesem Fall als das Problem der Art und des Umfanges der Reduktion der Theorie Luhmanns innerhalb der Literaturtheorie bzw. Kunstgeschichte formuliert. In der einführenden Studie zum Sammelband Systemtheorie der Literatur hat Literaturtheoretiker Jürgen Fohrmann hinzugefügt, dass die Reduktion der Theorie Luhmanns und ihre Operationalisierung innerhalb der Literaturwissenschaft keineswegs zufällige oder willkürliche Form hat: "Natürlich ließ sich nur lesen, was auf der Basis der schon bestehenden disziplinären Matrix lesbar war." (Fohrmann 1997: 7) Vergleichbar mit den Beispielen aus der Literaturwissenschaft sind zwei Studien, die die Rezeption der Theorie Luhmanns innerhalb der Theologie beobachtet haben (Dallmann 1994, Beyer 1996).

Die Frage nach der Form des Theorietransfers ist natürlich auch der Musikwissenschaft bekannt. Eines der letzten Beispiele ist die umfassende Studie des USamerikanischen Musikwissenschaftlers Adam Krims (Krims 1998) über die musikwissenschaftlichen Beobachtungen der Dekonstruktion. Seine eigene Beobachtung dieser Beobachtungen endet mit der Folgerung, die wohl vergleichbar mit der Fohrmanns ist: Er sieht in den musikwissenschaftlichen Versuchen, die Dekonstruktion an die Musikanalyse anzuwenden, gerade diejenige Reduktion der Theorie Derridas, die eine Bestätigung der schon bestehenden theoretischen Voraussetzungen der amerikanischen Musikwissenschaft möglich macht. Es ist allerdings hier hinzuzufügen, dass Krims' Beobachtung notwendigerweise auch mit bestimmten Voraussetzungen operiert, und zwar mit solchen, die konstitutiv für das vom Autor beobachtete, und im gewissen Sinn kritisierte, Wissenschaftsverständnis sind: Z. B. geht seine Beobàchtung von der Voraussetzung aus, dass ihr Gegenstand zum Sys- 
tem der US-amerikanischen historischen Musikwissenschaft gehört. Da dieses System auch der Ort ist, an dem Krims' Beobachtung sich selbst plaziert, muß sie die dort legitimen Formen der Musikforschung als Voraussetzung nehmen, auch (und besonders) dann, wenn sie eine Kritik an diesen Formen übt. Zu diesen Formen gehört z. B. Musikanalyse, besonders die Schenkerscher Prägung, die in den USA, wie bekannt, sogar einen institutionalisierten Rang traf. Krims' Beobachtung der musikwissenschaftlichen Derrida-Beobachtungen versucht zu zeigen, dass diese Beobachtungen keineswegs eine Abweichung von der bestehenden Matrix, die die Musikanalyse als eine rein formalistische Praxis darstellt, sind, sondern ganz im Gegensatz, dass sie diese Matrix nur weiter perpetuieren. Die Beobachtung, die Krims unternahm, sollte auch gewiße Kritik dieser Praxis sein. Allerdings könnte man sagen, dass diese Kritik sich selbst als keine totale Ablehnung des musikwissenschaftlichen Systems versteht, sondern vielmehr als ein Versuch, die bestehende Form der Musikanalyse zu erweitern und damit zu retten (zu dieser Position auch Krims 1998a). Es erscheint aber wichtig nicht zu vergessen, dass sowohl Krims' Beobachtung, als auch die erwähnten Beobachtungen in den anderen Disziplinen, immer innerhalb eines konkreten Wissenschaftssystems operieren, was auf jeden Fall ihre Vergleichbarkeit erschwert. Die Frage aber ist, wie Krims und andere erwähnte Autoren den Theorietransfer aus einer fremden in ihre eigene Disziplin beobachten, wenn sie gleichzeitig ein Teil der beobachteten Disziplin sind. Von dieser Frage wird später noch die Rede sein.

Dass das Programm der Interdisziplinarität auch die Frage nach der Identität der Disziplin impliziert, ist auch aus der Musikwissenschaft selbst bekannt. Einen der bekanntesten Fälle in der deutschen Musikwissenschaft stellt die Diskussion um die Kompetenz und Eigenständigkeit der systematischen Musikwissenschaft dar, die durch den Text Georg Feders (Feder 1980) angeregt wurde. Es handelt sich, wie bekannt, um einen Versuch, der systematischen Musikwissenschaft die Identität zu verneinen, und zwar aus der Position der historischen Musikwissenschaft. Die systematische Musikwissenschaft sei eine Disziplin, die nur die Methoden anderer Disziplinen übernimmt und ihre Resultate wiederholt, und deswegen seien ihre Identität und Eigenständigkeit fraglich. Es ist aber auch bekannt, dass gerade die theoretische Konstruktion dieses Versuchs in verschiedenen zeitgenössischen Theorietraditionen als Lieblingsbeispiel gilt, an dem möglich ist, die These von der Selbstwidersprüchlichkeit des substantialistischen Identitätsbegriffs plakativ zu demonstrieren.

Den Text von Hans-Peter Reinecke (Reinecke 1993) könnte man als eine mögliche Antwort auf Feders Versuch verstehen, und zwar nicht nur als Antwort auf die Frage, wie das Identitätsproblem innerhalb der Musikwissenschaft behandelt wird, sondern auch wie dieses Problem behandelt werden kann, wenn es um die äußeren Granzen der Musikwissenschaft als Disziplin geht. Der Vorschlag Reineckes ist einfach: Die Musikwissenschaft sollte nach außen greifen, um sich selbst zu sehen. In der Form einer Beobachtung zweiter Ordnung sollte sie sich selbst beobachten, um zu sehen, wie sie ihre eigene Gegenstände konstruiert hat (123). Auch in diesem Fall könnte man jenes Problem erkennen, das schon bei den Beo- 
bachtungen des Theorietransfers zu sehen ist: Von welcher Position aus ist es möglich die eigene Diziplin so zu sehen, als wäre man ein Außenstehender.

Einige Vorschläge gibt es schon: Z. B. Musikanthropologe Henry Kingsbury (in den Arbeiten Kingsbury 1988 und Kingsbury 1991) unternahm, ausgehend vom USamerikanischen System der Musikforschung, eine Beobachtung der amerikanischen Musikwissenschaft, die sich selbst innerhalb der Ethnomusicology plazierte. Allerdings ist zu vermuten, dass so ein Versuch nicht unbedingt als eine Beobachtung der Musikwissenschaft aus der Position der Musikwissenschaft selbst angesehen werden muß. Eher scheint, hinsichtlich der Lagen der Musikwissenschaft und Ethnomusikologie in den USA als soziale Systeme, dass es sich in diesem Fall um eine Fremdbeobachtung handelt, das heißt: Eine Beobachtung, die Musikwissenschaft von außen beobachtet. Eine ähnliche Position kann man aus den Text des schwedischen Ethnomusikologen Olle Edström entnehmen (Edström 1997), obwohl man dort das Verhältnis zwischen Ethnomusikologie und Musikwissenschaft als enger ansieht.

\section{Aufgabe, theoretischer Rahmen}

Der folgende Aufsatz soll auch eine Beobachtung zweiter Ordnung sein. Den Anregungen folgend, die von der Lektüre der hier erwähnten Texte hervorgerufen wurden, könnte man seine Aufgabe einerseits als eine Beobachtung eines konkreten Theorietransfers in die Musikwissenschaft bestimmen; andererseits wird sich mein Referat mit der Frage auseinandersetzen, wo diese Beobachtung stattfindet, bzw. wie man ihre Position bestimmen kann. Als konzeptueller Rahmen für diese Beobachtung der Musikwissenschaft ist die soziologische Systemtheorie Niklas Luhmanns ausgewählt worden und das konkrete Beispiel, das beobachtet wird, ist gerade die musikwissenschaftliche Beobachtung dieser Theorie.

Wenn es jetzt um die Frage nach der eigenen disziplinären Position handelt, scheint es, hinsichtlich des ausgewählten theoretischen Rahmens, dass die Antwort selbstverständlich ist: Da die Theorie Luhmanns eine soziologische Theorie ist, sollte die Beobachtung der Musikwissenschaft, die sich auf diese Theorie stützt, zu der Disziplin gehören, die als eine soziologische Beobachtung der Musik schon mehr oder weniger etabliert ist - nämlich der Musiksoziologie. Dass aber gerade die Position der Musiksoziologie als Disziplin überhaupt nicht so selbstverständlich ist, wie es scheint, kann man schon aus zwei neueren Selbstbeschreibungen dieser Disziplin entnehmen. So behauptet z. B. Christian Kaden im entsprechenden Artikel in der neuen Ausgabe der $M G G$ (Kaden 1997) schon im ersten Satz, dass die Musiksoziologie "ein (...) Arbeitsgebiet der Musikwissenschaft" sei (1618), bzw. dass sie "an der Grenze zwischen Historischer und Systematischer Musikwissenschaft" siedele (1620). Nach kurzer Darstellung der Themenkreise, die in den bisherigen musiksoziologischen Arbeiten vorkamen, formuliert Kaden ein kurzes Resüme: "Musiksoziologie widmet sich folglich nicht einem abstrakten Verhältnis von Musik und Gesellschaft (...). Sie begreift Musik selbst (...) als fait social." (1619) Wenn man aber an die von Krims unternommene Analyse der in den musikwissenschaftlichen Schriften häufig benutzten Formulation Musik selbst (Krims 1998) denkt, könnte 
man sagen, dass Kaden gerade dort als Aufgabe der Musiksoziologie die musikwissenschaftliche Beschäftigung mit der Struktur der Musik postuliert.

Es ist natürlich nicht die Frage, ob Kaden recht hat oder nicht. Wichtig ist, dass seine Auswahl bestimmte Folgen hat: Da er die Aufgabe der Musiksoziologie so normativ bestimmt hat, muß er alle anderen Positionen als gewiße Abweichungen von der Norm behandeln. Dieses Problem kann man besonders klar sehen, wenn Kaden die Geschichte der Musiksoziologie als ausdifferenzierte Disziplin darstellt: Alles läuft relativ glatt bis zum bekannten Streit zwischen Adorno und Silbermann. In diesem Moment führt Kaden eine neue Opposition ein, um zu zeigen, dass es in diesem Streit eine von ihm bevorzugte Seite gibt, nämlich die Adornos, die sich zur Aufgabe der eigenen Untersuchung, "wie Gesellschaft in Musik erscheint, wie sie aus der Textur herauszulesen ist" (Adorno 1962: 225), gemacht hat. Natürlich gibt es noch eine andere Seite, die ihre Aufmerksamkeit nicht auf Musik selbst richtet, und die deswegen keine echte Musiksoziologie sei, sondern bloßer "Anwendungsbereich der allgemeinen Soziologie (Kaden 1997: 1623). Die erste Seite der Unterscheidung bezeichnet Kaden als Musiksoziologie, und die zweite als Soziologie der Musik. Dieser Unterscheidung folgend, muß er notwendigerweise alle Positionen, die mit keinem musikwissenschaftlichen Konzept der Musik selbst rechnen, aus der Musiksoziologie ausgrenzen. Sofort bekommt er unerwünschte Probleme: Gehören dann zur Musiksoziologie etwa auch (für Kaden selbstverständlich) die bekannten Schriften von Georg Simmel (Simmel 1975) und Max Weber (Weber 1921)? Hier ist z. B. an den Aufsatz des Soziologen Horst Jürgen Helle (Helle 1992) zu denken, in dem er die These vertritt, dass die Soziologen Simmel und Weber sich nicht mit der Musik beschäftigen, um die musikwissenschaftlichen Interesse an $\mathrm{Mu}$ sik selbst zu treiben, sondern weil sie eigene soziologische Theorien am Beispiel der Musik besonders plausibel demonstrieren können (133). So können ihre Schriften, die Kaden als Anfang der Musiksoziologie als eine ausdifferenzierte wissenschaftliche Disziplin bezeichnet, auch als Beispiele gerade für einen Anwendungsbereich der allgemeinen Soziologie angesehen werden. Es stellt sich allerdings die grundsätzliche Frage, ob überhaupt die Musik selbst als privilegiertes Interesse der Musikwissenschaft gelten muß, wie das aus dem Kaden-Entwurf zu entnehmen ist.

Das alles könnte aber noch ein wenig ausdifferenziert werden. Es ist nämlich zu bemerken, dass Kaden die erwähnten Schriften von Simmel und Weber doch von den musikwissenschaftlichen Beobachtungen unterscheidet, wenn er später gerade den Musikwissenschaftlern eine "Selbsteinschnürung" (1622) vorwirft, weil sie die Schriften von großen Soziologen nicht genug rezipierten. Allerdings könnte man auch vermuten, dass Kadens Entwurf mit der Voraussetzung rechnet, nach der die Musiksoziologie seit Simmel und Weber sich soweit ausdifferenziert hat, dass sie später nicht mehr einem soziologischen Interesse dienen sollte. Es scheint, dass soziologische Arbeiten über Musik, die nach Weber entstanden sind, für die von Kaden konstruierte Musiksoziologie nicht mehr von besonderem Interesse sind, weil sie den normativen Anspruch dieser Musiksoziologie nicht erfüllen können. 
Im gleichen Jahr ist auch die Dissertation der Soziologin Katharina Inhetveen erschienen (Inhetveen 1997), in der die Musiksoziologie als Disziplin anders als bei Kaden dargestellt ist. Es handelt sich hier um eine Untersuchung "der musiksoziologischen Forschung innerhalb der Disziplin Soziologie." (9) Wenn man sich aber fragt, wie sie dann jene musiksoziologische Arbeiten behandelt, die aus der Position der Musikwissenschaft geschrieben sind, scheint die Antwort nicht besonders klar. Im Unterschied zu Kaden, scheint es, dass sie doch keinen normativen Anspruch an die Zugehörigkeit zur Musiksoziologie gestellt hat, da sie am Rande auch einige musiksoziologischen Arbeiten analysiert, die vom musikwissenschaftlichen Standpunkt ausgehen. Obwohl schon vom Rahmen der Untersuchung ausgeschloßen, werden diese Arbeiten doch als Beiträge zur Musiksoziologie gesehen. Es scheint aber bei einem differenzierteren Lesen, dass Inhetveen doch mit einem Kriterium für die Zugehörigkeit zur Musiksoziologie operiert, welches nicht unbedingt die formale soziologische Ausbildung als Voraussetzung haben muß: Die fachsoziologisch ausreichende Argumentationsweise. Insofern ist ihre Position vergleichbar mit jener von Alphons Silbermann, die als Konstruktion der "Pole der Musiksoziologie" (Silbermann 1958, Silbermann 1963) bekannt ist. Diese Konstruktion, die gegen den von Silbermann formulierten Monopolanspruch der Musikwissenschaft auf alle wissenschaftliche Erforschung der Musik entworfen wurde, erachtet eine Zusammenarbeit von Soziologie und Musikwissenschaft als wünschenswert. Deswegen schreibt Silbermann vor, dass Wissenschaftlerinnen und Wissenschaftler für musiksoziologische Forschungen sowohl soziologisches als auch musikwissenschaftliches Fachwissen beherrschen sollten. Da Inhetveen aber die soziologische Musiksoziologie für quantitativ schwächer entwickelt hält als die musikwissenschaftliche Musiksoziologie, sieht sie die Schwierigkeit gerade in der ungenügenden musikalischen Ausbildung der Fachsoziologen: "Wird nun davon ausgegangen, dass Musiksoziologie musikologisches Fachwissen erfordet, so hat vielleicht auch die Geschichte der Disziplin dazu beigetragen, dass sie sich innerhalb der Soziologie kaum etabliert hat." (Inhetveen 1997: 46) Man kann sich aber fragen, warum Soziologie gerade das musikwissenschaftliche Wissen als einziges mögliches Wissen über Musik anerkennen sollte? Die Antwort auf diese Frage könnte man vielleicht in der Unterscheidung alltägliches Wissen/wissenschaftliches Wissen finden, mit der Inhetveens Beobachtung operiert. So behauptet die Autorin, anschließend an die Unterscheidung von Susanne Langer, dass das alltägliche Wissen über Musik ähnlich dem mythischen Denken funktioniert, während das wissenschaftliche Wissen anders ist: "Wissenschaft kann zwar den nicht-diskursiven Gehalt von Musik auch nicht formulieren, sie kann sich aber mit den symbolischen Formen als solchen befassen, getrennt von dem durch sie Vermittelten. Musiktheorie, systematische und historische Musikwissenschaft stellt das Werkzeug für eine Annäherung an die Gestalt dieser Symbole. Ohne musikologisches Fachwissen kann eine Abstrahierung der Symbole vom Gegenstand kaum stattfinden, da ihre äußere Struktur nicht erfaßt werden und damit die Trennlinie zum Repräsentierten nicht gezogen werden kann. (...) Wenn sich alltäglicher und wissenschaftlicher Umgang mit Musik in dieser Weise unterscheiden, bedeutet das, dass wissenschaftliche Beschäftigung mit Musik auf 
einer anderen Ebene stattfindet als allägliche, und dass sie ausführliches Wissen über den Gegenstand erfordert, das eben im Alltag nicht erworben werden kann. Es scheint, dass dies die Musiksoziologie von anderen Bindestrich-Soziologien unterscheidet: Der alltägliche Umgang mit Politik, Religion oder Geschichte ist zwar nicht unbedingt wissenschaftlich, er spielt sich aber nicht auf einer völlig anderen, nicht einmal diskursiven Ebene ab, wie es bei Musik weitgehend der Fall ist." (45/46) Sogar die Entscheidung, dass musikwissenschaftliches Wissen das wahre Wissen über Musik sei, versucht sie zu rechtfertigen, und zwar mit der These über den besonderen "Kunstcharakter von Musik" (44). Man könnte aber diese Rechfertigung auch umkehren, um zu sehen, dass gerade postulierte Besonderheit der Musik als ein Konstrukt der musikwissenschaftlichen Beobachtungen angesehen werden könnte, die man als wahres Wissen über Musik hält. Wenn man aber schon das Alltagswissen aus der Wissenschaft ausgrenzt, so bedeutet das noch lange nicht, dass man unbedingt die erste Seite der weiteren möglichen Unterscheidung musikwissenschaftliches wissenschaftliches Wissen/nichtmusikwissenschaftliches wissenschaftliches Wissen auswählen muß: Es ist denkbar, dass das Wissen über Musik, die im Rahmen von Soziologie oder Psychologie getrieben wird, auch als relevantes wissenschaftliches Wissen über Musik angesehen werden kann. Es scheint, dass gerade das Postulat vom musikwissenschaftlichen Wissen als das angeblich einzige angemessene Wissen über Musik nur weiter den von Silbermann (und später auch vom Soziologen Frank Rotter, z. B. in Rotter 1992) beklagten Monopolanspruch der Musikwissenschaft reproduziert. Die Tendenz innerhalb der Soziologie, dass man die musikwissenschaftliche Beobachtung der Musik gegenüber den anderen Beobachtungen bevorzugt, hat allerdings schon eine gewisse Tradition: Man sollte z. B. auf die Studie von Christoph Braun hinweisen (Braun 1992), die eine von Max Weber unternommene Rezeption der damaligen musikwissenschaftlichen Literatur zu rekonstruieren versuchte.

So scheint, dass Ihnetveen am Ende das Verhältnis zwischen den beiden Polen der Musiksoziologie analog zur Unterscheidung Selbstbeschreibung/ Fremdbeschreibung konstruiert, wenn sie schreibt: "Der genuin soziologische Anteil an der musiksoziologischen Literatur, verfaßt von Autoren und Autorinnen mit fachlicher Ausbildung in Soziologie, scheint gering, vor allem im Verhältnis zu den musikwissenschaftlichen Beiträgen (...) - kleiner wohl als in anderen Teilgebieten der Soziologie. Es würde sicher Verwunderung hervorrufen, wenn ein Großteil etwa der Arbeiten zur Industriesoziologie verfaßt wäre von Volks- und Betriebswirten, Schlossern und Verfahrestechnikern - von denen die meisten auch ohne soziologische Ausbildung seien." (Inhetveen 1997: 43/44) Das entsprechende Verhältnis vergleicht Rotter mit dem zwischen Rechtstheorie und Rechtssoziologie, und zwar so, "dass Rechtstheorie und Rechtsdogmatik als Formen der Selbstbeschreibung des Rechtssystems gefaßt werden, während die Rechtssoziologie das System von außen beobachtet und beschreibt und dadurch mehr, aber auch weniger sieht als die Rechtstheorie (...)." (Rotter 1992: 89) Man könnte aber sagen, dass auch diese Konstruktion, nach der soziologische Beschreibung sozusagen eine Beschreibung zweiter Ordnung sei, schon eine Tradition innerhalb der Soziologie hat. Schon Sim- 
mel in seinem berühmten Entwurf aus 1890 hat der Soziologie als Wissenschaft eine ähnliche Stelle gegeben: "Sie ist eine eklektische Wissenschaft, insofern die Produkte anderer Wissenschaften ihr Material bilden. Sie verfährt mit den Ergebnissen der Geschichtsforschung, der Anthropologie, der Statistik, der Psychologie wie mit Halbprodukten; sie wendet sich nicht unmittelbar an das primitive Material, das andere Wissenschaften bearbeiten, sondern, als Wissenschaft sozusagen zweiter Potenz, schafft sie neue Synthesen aus dem, was für jene schon Synthese ist." (Simmel 1989: 116)

Es ist sicher möglich, nach so skizzierter Diskussion, sich auch die Frage zu stellen, ob die Position, von der aus man eine Beobachtung unternimmt, so wichtig ist? Ist es entscheidend, ob man Musik von der musikwissenschaftlichen oder von der soziologischen Seite beobachtet, selbst dann, wenn man wie Inhetveen die soziologische Beobachtung als eine Beobachtung sieht, die weitgehend von der musikwissenschaftlichen Beobachtung abhängt?

Wenn man im konzeptuellen Rahmen der Theorie Luhmanns zu argumentieren versucht, würde man sagen, dass gerade die Positionierung der Beobachtung als entscheidend angesehen werden sollte, weil sie sowohl die Fragen als auch die Antworten als keine Gegebenheiten sieht, sondern als Konstrukte, die ein Beobachter ausgehend von einer Differenz (bzw. einer Form) konstruiert. Da nach Luhmann zwei verschiedene wissenschaftliche Disziplinen zwei verschiedene Formen benutzen, um ihre eigene Kommunikationen zu codieren und in dieser Weise von anderen zu unterscheiden, könnte man auch sagen, dass Soziologie und Musikwissenschaft ihre eigenen Gegenstände anders voneinander konstruieren, weil sie mit zwei voneinander verschiedenen Codes operieren.

Bevor man aber den Transfer der Systemtheorie Luhmanns in die Musikwissenschaft diskutiert, sollte man vielleicht kurz skizzieren, wie man selbst diese Theorie versteht. Die Darstellung kann leider in diesem Moment nicht besonders differenziert sein, weil die Zeit einfach zu knapp ist; deswegen wird sie sich auf jene Beobachtungen beschränken, die notwendig für die weitere Argumentation scheinen.

Um einen systemtheoretischen Trick zu benutzen, könnte diese Theorie dargestellt werden, indem man ihre eigene Evolution als einen Differenzierungsprozeß von anderen theoretischen Entwürfen beschreibt. So kann man frühere Arbeiten von Luhmann, seit dem in 1964 veröffentlichte Buch Funktionen und Folgen formaler Organisation, als Anknüpfungen an die Tradition des Funktionalismus sehen, jene theoretische Tradition innerhalb der Soziologie, deren Konzeptualisierung der Gesellschaft man häufig differenziell zur marxistischen Tradition (einschließlich der Tradition der Kritischen Theorie) sieht. Wenn man weitere Differenzen angeben möchte, könnte man z. B. auf die Anmerkung des Soziologen Dirk Baecker hinweisen, dass Luhmanns Position sowohl gegen kritische als auch gegen positive Soziologie gerichtet ist (Baecker 1999: 35). In diesem Sinn schreibt der Soziologe Daniel Barben, dass Luhmanns Systemtheorie sich innerhalb der deutschen Soziologie "in einer doppelten Frontstellung (Barben 1997: 41) sowohl gegen die Frankfurter als auch gegen die Kölner Schule richtet. Luhmanns soziologische Theorie wird von Baecker als eine reflexive Soziologie (Baecker 1999: 36) bezeichnet, "die 


\section{MUZIKOLOŠKI ZBORNIK • MUSICOLOGICAL ANNUAL XXXV}

strikt auf sich selbst zurückkommt, wenn sie anderes beobachtet, und die genau diese Bewegung auch ihrem Gegenstand unterstellt." (45/46) Die Skizze einer Geschichte der Theorie Luhmanns, die von Barben stammt, sieht in den Siebzigern als weiteren Differenzierungsschritt Luhmanns Reformulierung des Funktionalismus seines Lehrers Talcott Parsons, und zwar so, dass die "Strukturen (...) nun nicht mehr als gegeben vorausgesetzt und auf ihre Funktion gefragt, sondern, im Hinblick auf bestimmte Funktionen, als austauschbar behandelt (wurden)." (Barben 1997: 43). Nach Barben fungiert als letzter Bezugspunkt funktionaler Analysen in Luhmanns Texten aus dieser Zeit das Problem der Komplexität (44).

1971 fand der bekannte Streit zwischen Luhmann einerseits und Jürgen Habermas andererseits statt, der in einem Sammelband (Habermas/Luhmann 1971) dokumentiert wurde und der viele weitere kommunikative Anknüpfungen angeregt hat. Die Diskussion began Habermas, mit dem Vorwurf, die Systemtheorie Luhmanns sei eine "neue Form der Ideologie" (Habermas 1971: 239). Es gab mehrere Streitpunkte, aber die wichtigsten scheinen das Problem der Ideologie bzw. der Ideologiekritik und das Problem der Normativität der Theorie zu sein. Habermas sieht den Ideologiebegriff in der Tradition der Kritischen Theorie, als einen Differenzbegriff zum Wahrheitsbegriff, und zwar so, dass man unter der Wahrheit "die Vernünftigkeit einer in zwangloser und uneingeschränkter Kommunikation erreichbaren Verständigung " (241) auffaßt. Luhmann knüpft dagegen die Wahrheit nicht an den Konsens, sondern ist der Meinung, dass man eine abstraktere Fassung des Wahrheitsbegriffs braucht, um die Selbstwidersprüche der normativen Kommunikationstheorien zu vermeiden. Er schreibt: "Habermas definiert Wahrheit durch ein idealisiertes Verständnis von Intersubjektivität und meint, so und nur so die Wahrheitsfähigkeit praktischer Geltungsansprüche begründen zu können. Dieser Zusammenhang ist für mich nicht einsichtig; ich sehe nicht, wie man anders als durch Äquivokationen und unmerkliche Begriffsverschiebungen von einem idealisierten Wahrheitsbegriff zu einer normativen Geltung idealer Sprechsituationen und von dort aus zur Wahrheitsfähigkeit praktischer Fragen kommen kann. (...) Außerdem liegt in der definitorischen Einführung von Idealisierungen der Verzicht darauf, aus einem vorgelagerten begrifflichen Bezugsrahmen eine Begründung und eine Richtungskontrolle für die idealisierenden Bestimmungen $\mathrm{zu}$ gewinnen. Deshalb möchte ich versuchen, Wahrheit im Kontext einer allgemeinen Theorie der Kommunikationsmedien funktional zu definieren, um dann im Rahmen dieser Theorie erstens Sinn und Funktion von Idealisierungen, zweitens die Funktion von Normierungen als Elementen des Wahrheits-codes und drittens den Unterschied zu anderen Kommunikationsmedien, namentlich zu Macht, begründen zu können." (Luhmann 1971: 343/344) Um so mehr zu Macht, weil sie, Luhmanns Meinung nach, als das Kommunikationsmedium des politischen Systems dient. Ideologiekritische Einwände gegen die Systemtheorie, die Habermas unternimmt, sieht er nämlich gerade als politische: "Den nicht unberechtigten Vorwurf, die begrifflichen und methodischen Unzulänglichkeiten einer Theorie der Gesellschaft zu überspielen, gebe ich Habermas zurück; nur dass er die wissenschaftliche Not nicht in die 
Tugend eines pragmatischen Vorgehens, sondern in die Tugend politischer Diskussion übersetzt." (404)

Weitere Differenzierung der Systemtheorie Luhmanns könnte man mit Barben als einen Übergang zur Theorie selbstreferentieller Systeme verstehen, den man vom Anfang der Achtziger schriftlich folgen kann. Die entscheidende Operation innerhalb dieses Übergangs sieht er im Transfer des Autopoiesisbegriffs aus der Biologie in die soziologische Theorie. Diesen Schritt hat Barben detailhaft untersucht, und zwar in Hinsicht auf einige verwandten Versuche innerhalb der Soziologie einerseits und andererseits hinsichtlich der Theorien der Sozialität, die von Biologen Humberto Maturana und Francisco Varela selbst entworfen wurden (ein Beispiel ist der Text Maturana 1996). Die Differenz zwischen den Theorien der Sozialität, die aus der Biologie kommen, und der Theorie Luhmanns, sieht Barben schon auf der Elementarebene: Während Maturana "soziale Systeme von den Individuen her (denkt)" (Barben 1997: 36), fungieren für Luhmann Kommunikationen als konstitutive Elemente der Gesellschaft. Seine Auflösung des Menschenbegriffs in voneinander operativ getrennten und geschloßenen organischen, psychischen und sozialen Systemen ist übrigens immer noch sehr häufig ein Streitpunkt, wenn es um die Diskussion über Luhmanns Theorie geht. Luhmann selbst hat mehrmals versucht, seine eigene Position zu verteidigen (ein Beispiel kann man im Beitrag Luhmann 1994 finden; diesem Thema sind auch Beiträge in Luhmann 1996 gewidmet). Auf der anderen Seite kann man gerade in diesem theoretischen Schritt jene Entscheidung sehen, aus deren Konsequenzen einige systemtheoretischen Ansätze ihre Fruchtbarkeit schöpfen: Z. B. die Arbeiten des Soziologen Peter Fuchs stellen, nach Wolfgang Ludwig Schneider, gerade einen Versuch dar, diese Konsequenzen systematisch zu entfalten (Schneider 1998: 198).

Die zweite Differenz, von der Barben spricht, unterscheidet nicht die Theorie Luhmanns von den biologischen Theorien der Sozialiät, sondern von den anderen soziologischen Theorien, die biologischen Autopoiesisbegriff parallel mit Luhmann in die Soziologie importiert haben. Diese Differenz sieht Barben als jene, die zwischen zwei unterschiedlichen Strategien zu sehen ist. Die erste, deren Hauptvertreter Soziologe Peter Hejl sei, bezeichnet er als "Aufbau-Strategie", während die zweite "Analogisierungsstrategie" (37/38) genannt wird, und deren Vertreter Luhmann sei. "Die eine knüpft direkt an die biologischen Begriffsbestimmungen, ihren materialen Bedeutungsgehalt an. Beim Versuch, konzeptionell zu einer Theorie autopoietischer sozialer Systeme aufzusteigen, fungiert die Theorie autopoietischer biologischer Systeme als epistemologische Richtschnur. So werden die biologischen Begriffe nur dann zur Übertragung in ein anderes Feld freigegeben, wenn auch in diesem dieselben als essentiell erachteten Bedeutungsaspekte vorliegen. Andernfalls wird nach verwandten, angrenzenden Begriffen gesucht, die eine Ausdehnung bzw. Generalisierung ermöglichen. (...) Bei der anderen Strategie wird die Begrifflichkeit autopoietischer Systeme formal und abstrakt aus der Biologie übernommen, um sie dann in sozialwissenschaftliches Terrain einzuarbeiten. So wird der Ansatz zurückgewiesen, die Theorie sozialer Systeme auf die Theorie autopoietischer biologischer Systeme aufzubauen. Dadurch entfallen komplizierte Vermit- 
tlungsschritte, wozu auch die Klärung von Fragen der Isomorphie zwischen den Ebenen gehört. (...) In diesem Vorgehen der terminologischen Übertragung kann dieselbe Begrifflichkeit in verschiedenen Bereichen mit ebenso verschiedenen Bedeutungen auftauchen - so dass sich also unter denselben Worten ganz unterschiedliche Begriffe verbergen können." (37/38) Während bei der Aufbau-Strategie zusammen mit den biologischen Begriffen auch die von den erwähnten Biologen vertretene Vorstellung der Gesellschaft übernommen wird, ist es im anderen Fall möglich, eine Theorie so zu konstruieren, dass sie gewiße theoretische Probleme innerhalb der Soziologie elegant lösen kann. Das bedeutet vor allem, dass man auf diese Weise die Normativität der Theorie vermeiden möchte: Mit dieser Strategie, könnte man sagen, sollten die Behauptungen, wie z. B. jene, dass "soziales Verhalten auf Kooperation (basiert), nicht auf Kampf" (Maturana 1996: 300), vermieden werden, oder zumindest schon als Produkte einer umfassenderen und abstrakteren Operationsweise. Diese Differenz scheint insofern wichtig, weil sie gleichzeitig als eine Markierung dienen könnte, die man braucht, wenn man die Position Luhmanns in Hinsicht auf die Vertreter (oder: andere Vertreter) des Radikalen Konstruktivismus beobachten möchte.

Die ersten Schritte Luhmanns in die Richtung der Theorie selbstrefferentieller Systeme folgt das Buch Soziale Systeme (Luhmann 1984), das häufig als sein Hauptwerk angesehen wird. Dort ist Luhmanns Variante der Systemtheorie als eine allgemeine Theorie dargestellt, die als einen Gegenentwurf zur drei Jahre früher veröffentlichen Theorie des kommunikativen Handelns von Habermas (Habermas 1981) gelesen werden kann (und häufig auch gelesen wird). Im Unterschied zu Habermas, der sich auf die Begriffe des Handelns, der Intersubjektivität und des Konsens' beruft, und insofern eine lange Tradition innerhalb der Soziologie und Philosophie folgt, beruft sich Luhmann eher auf die theoretischen Ansätze aus der Kybernetik, Biologie oder Kommunikationswissenschaft, um die erwähnten soziologischen Konzepte schon als kommunikative Konstrukte und damit keineswegs als verpflichtende Normen darzustellen: "Sozialität ist kein besonderer Fall von Handlung, sondern Handlung wird in sozialen Systemen über Kommunikation und Attribution konstituiert als eine Reduktion der Komplexität, als unerläßliche Selbstsimplifikation des Systems." (Luhmann 1984: 191) Kommunikation sei aber keine Übertragung, sondern ein "Prozessieren von Selektionen" (194), und zwar ein dreistelliges: "Es geht nicht um Absendung und Empfang mit jeweils selektiver Aufmerksamkeit; vielmehr ist die Selektivität der Information selbst ein Moment des Kommunikationsprozesses. (...) Entscheidend ist, dass die dritte Selektion sich auf eine Unterscheidung stützen kann, nämlich auf die Unterscheidung der Information von ihrer Mitteilung." (194/195) So ist Kommunikation als ein konstruktiver Prozeß entworfen, in dem Alter und Ego, jedes für sich selbst, eigene kommunikativen Selektionen prozessieren, und zwar so, dass eine Differenz zwischen ihnen nie aufgehoben werden kann. Wenn dieses der Fall ist, wie in der Theorie der Intersubjektivität, könnte man, nach Fuchs, schon über die "Ätherhypothese der Soziologie" (Fuchs 1993: 19) sprechen. 
Der Differenz könnte man weiter folgen. So konzeptualisieren Habermas und Luhmann den Systembegriff selbst unterschiedlich: Während Habermas unter diesem Begriff einen konsensuell eingespielten Handlungsrahmen versteht, sieht Luhmann das System immer differenziell zur Umwelt: "Das zentrale Paradigma der neueren Systemtheorie heißt 'System und Umwelt'. Entsprechend beziehen sich der Funktionsbegriff und die funktionale Analyse nicht auf 'das System' (...), sondern auf das Verhältnis von System und Umwelt. (...) Der Ausgangspunkt aller daran anschließenden systemtheoretischen Forschungen ist daher nicht eine Identität, sondern eine Differenz. (...) Alles, was vorkommt, ist immer zugleich zugehörig zu einem System (oder zu mehreren Systemen) und zugehörig zur Umwelt anderer Systeme." (Luhmann 1984: 242/243) Die Systeme sind bei Luhmann operativ geschloßen und - wenn es sich um psychische und soziale Systeme handelt - reproduzieren sie sich autopoietisch im Medium Sinn.

Es scheint hier wichtig, noch zwei weitere Konzepte von Luhmann zu erwähnen, weil sie in der folgenden Argumentation eine Rolle spielen werden: Die Differenzen Operation/Beobachtung und Beobachtung erster und zweiter Ordnung. Den Beobachtungsbegriff könnte man, mit der Soziologin Elena Esposito, als "eine spezifische Operationsweise" verstehen, "die eine Unterscheidung benutzt, um die eine oder andere Seite der Unterscheidung zu bezeichnen. (...) Auch die Beobachtung ist jedoch ihrerseits eine Operation eines Systems und als solche der eigenen Reproduktion gegenüber ebenso blind: Die Anfangsunterscheidung ist ihr blinder Fleck, das heißt der Punkt, den sie nicht beobachten kann." (Esposito 1998: 124/125) Der Unterscheidung zwischen Beobachtung erster und zweiter Ordnung, die Luhmann von dem Kybernetiker Heinz von Foerster und dem Anthropologen Gregory Bateson übernommen hat, widmete er in seinem Buch über Kunst ein ganzes Kapitel. Dort schreib er: "Jede Beobachtung, auch die Beobachtung zweiter Ordnung, benutzt eine Unterscheidung, um die eine (aber nicht die andere) Seite zu bezeichnen. (...) Als Beobachtung zweiter Ordnung wollen wir die Beobachtung von Beobachtungen bezeichnen. Auch die Beobachtung zweiter Ordnung ist demnach als Operation eine Beobachtung erster Ordnung, nämlich die Beobachtung von etwas, was man als Beobachtung unterscheiden kann." (Luhmann 1995: 94) Das Beobachten zweiter Ordnung, schreibt Luhmann weiter, "hat, auf seine Wirkungen hin beobachtet, offenbar toxische Qualität. Es verändert den unmittelbaren Weltkontakt." (156) Damit ist aber nicht gesagt, dass die Beobachtung zweiter Ordnung einer Kritik gleicht: "Gewiß soll den Kritikern das Wort nicht abgeschnitten werden, und es geht auch nicht um eine in sich paradoxe Kritik des Kritisierens. (...) Es sollte nur eine Möglichkeit der Beobachtung zweiter Ordnung darübergelegt werden, damit man fragen kann, mit welchen Unterscheidungen Kritiker arbeiten, und warum gerade mit diesen und nicht mit anderen." (163/164)

Die weiteren Arbeiten Luhmanns nach seinem Buch Soziale Systeme von 1984 haben, so Barben, die dort entworfene theoretische Konstruktion nicht bedeutend verändert, obwohl es gewiße Umakzentuierungen gab. Sein Interesse richtet sich mehr auf besondere Funktionalsysteme der Gesellschaft, wie Wirtschaft, Wissenschaft, Recht und Kunst. 
Neben der Abgrenzungen von Habermas kann man auch die Auseinandersetzungen mit den (anderen) Konstruktivisten und die Abgrenzung von ihnen in manchen Arbeiten Luhmanns aus den Achtzigern finden; einige von ihnen sind im fünften Band aus der Reihe Soziologische Aufklärung (Luhmann 1990a) nachgedruckt (hilfreich bei diesem Problem sind auch die Unterscheidungen, die der Soziologe Armin Nassehi in seinem Text Nassehi 1992 beschrieben hat). Da die Arbeiten des Literaturwissenschaftlers Siegfried J. Schmidt auch für die musikwissenschaftlichen Beobachtungen eine Rolle spielen, wird jetzt aus der ganzen Diskussion zwischen Luhmann und anderen Radikalen Konstruktivisten nur das Verhältnis zwischen ihm und Schmidt kurz skizziert. Etwas früher als die erste Ausgabe von Habermas' Theorie des kommunikativen Handelns ist Schmidts Buch Grundriß der empirischen Literaturwissenschaft (Schmidt 1991) erschienen, in dem der Autor auch mit dem Systembergiff operiert. Doch die Systeme Schmidts scheinen wesentlich unterschiedlich vom Systembegriff aus den Sozialen Systemen Luhmanns: Ähnlich wie Habermas sieht Schmidt soziale Systeme als intersubjektiv-konsensuell eingespielte Handlungssysteme, deren Komponenten menschliche Handlungen sind. Dieser Konzeption, die dann auch die Begriffe wie Subjekt, Individuum oder eine allgemein humanistische Rhetorik voraussetzt, blieb Schmidt wesentlich treu auch in den Arbeiten, die schon unter dem Titel Radikaler Konstruktivismus stehen (z. B. in Schmidt 1996). In den Arbeiten Schmidts aus den Neunzigern kann man seine Versuche beobachten, sich immer wieder mit der Theorie Luhmanns auseinanderzusetzen und einige Konzepte aus ihr zu übernehmen. So ist seine Besprechung der Systemtheorie Luhmanns im Buch Schmidt 1994 noch relativ ablehnend in Hinsicht auf einige systemtheoretischen Konzepte. Er kritisiert Luhmanns Konzeption operativer Geschloßenheit der Systeme, seine scharfe Trennung sozialer von psychischer Systeme, und findet seinen Kommunikationsbegriff unhaltbar, weil dieser mit keinem Menschenbegriff mehr rechnet: Während Luhmann Handlungen nur für Teile der Kommunikation hält, bezeichnen für Schmidt Kommunikationsund Handlungsbegriff das Gleiche, weil Schmidt unter der Kommunikation eine gerade menschliche Handlung versteht. Luhmanns Konzept sei unempirisch, und das findet Schmidt nicht akzeptabel. Im Aufsatz Schmidt 1995 unternahm er eine andere Strategie (vielleicht auch unter dem Einfluß der heftigen Diskussionen über seinen früheren Entwurf, die man z. B. im Sammelband Barsch/Rusch/Viehoff 1994 finden kann): In diesem Text hat er das Konzept der Beobachtung zweiter Ordnung von Luhmann übernommen, um die Theorie Luhmanns selbst dieser Beobachtung zu unterwerfen. So hat er versucht, die Systemtheorie Luhmanns aus der Sicht der allgemeinen Systemtheorie zu beobachten, um zu sehen, wie Luhmann seine eigene Theorieentscheidungen getroffen hat. Aus dieser Beobachtung der Theoriearchitektur Luhmanns hat Schmidt am Ende herausgefunden, dass Luhmann seine eigene Theorie so konstruierte, dass er Systeme notwendigerweise für homogen halten muß, während Schmidt findet, dass es auch eine von Luhmann ausgeschloßene Möglichkeit gibt, nämlich die, die Systeme für heterogen zu halten (Schmidt 1995: 237). Das bedeutet konkret: Für Luhmann bestehen soziale Systeme immer nur aus den Kommunikationen, während Schmidt behauptet, dass sie auch andere Kompo- 
nenten haben können, wie z. B. Handlungen. In seinem letzten Buch von 1998 hat Schmidt sich noch mehr auf Luhmann gestützt: Jetzt behauptet er, dass seine Theorie nicht mehr mit den Subjekt- und Intersubjektivitätsbegriff operiert, oder zumindest nicht so normativ wie früher. Jetzt wendet er sich von analytischer und kritischrationalistischer normativer Grundlegung der Wissenschaft ab, und plädiert für die systemtheoretische Konstruktion der Wissenschaftstheorie als autopoietisches System, das aus kommunikativen Anknüpfungen besteht. Doch sind einige feine Unterschiede geblieben, in der ersten Reihe jene zwischen Luhmanns homogener Konzeption des Systems und seinen eigenen Entwurf des heterogenen Systems (Schmidt 1998: 186).

Diese Unterschiede in der Theoriedifferenzierung bei Luhmann selbst, und auch bei seinen Opponenten, könnten jetzt als eine Form im systemtheoretischen Sinn benutzt werden, um die musikwissenschaftlichen Beobachtungen zu beobachten. Es scheint in diesem Moment noch wichtig, kurz die Position Luhmanns zu skizzieren, wenn es um die Beobachtung der Wissenschaft geht. Er knüpft nämlich in diesem Fall an die Tradition der Wissenssoziologie, obwohl er gleichzeitig von dieser Tradition einigermaßen abweicht. Die Wissenssoziologie, wie sie von Luhmann konstruiert ist, soll eine soziologische Beobachtung zweiter Ordnung sein, die nicht mehr mit jenem Grundproblem zu tun haben sollte, das Luhmann als "die Vorstellung einer repräsentationalen Funktion" (Luhmann 1999: 155) des Wissens bestimmt. Statt der Vorstellung, dass "das Wissen sich auf die Außenwelt (bezieht)" (155), weiterzutreiben, versucht Luhmann das Problem der Wissenssoziologie abstrakter zu formulieren: "Alles Wissen ist also letztendlich Paradoxiemanagement, und dies in der Weise, dass man eine Unterscheidung vorschlägt, deren Einheit nicht thematisiert wird, weil dies das Beobachten in die Form einer Paradoxie bringen, also blockieren würde. (...) Angesichts dieses Befundes einer paradigmatischen Revolution in der Erkenntnistheorie hat die Wissenssoziologie zwei Möglichkeiten. Sie kann im Stile der klassischen Wissenssoziologie fortfahren und, mit veränderten Variablen, nach Zusammenhängen zwischen der Struktur der modernen Gesellschaft und dieser Typik von Kognitionstheorie fragen. Aber sie kann auch versuchen, den angebotenen Wissensbegriff zu übernehmen und sich selbst als konstruierte, also dekonstruierbare Selbstbeschreibung der Gesellschaft darzustellen." (173/174) Luhmann selbst hat sich für die zweite Möglichkeit entschieden: Die Wissenssoziologie sollte nicht mehr nach der Position des Menschen innerhalb der gesellschaftlichen Struktur fragen, um dann an diese Position seine Denkweise zuzuordnen (wie das im klassischen Konzept von Karl Mannheim der Fall ist, z. B. in Mannheim 1965), sondern sollte fragen, "welche Formen, welche Identitäten, welche Unterscheidungen man benutzt, um Paradoxien zu entfalten und, wie sprunghaft immer, durch operationsfähige Konzepte zu ersetzen." (Luhmann 1999: 176)

\section{Beispiele}

Obwohl es auf dem ersten Blick anders scheinen könnte, gibt es schon jetzt relativ viele musikbezogene Arbeiten, die sich auf die Systemtheorie Luhmanns stützen. 
Da hier nur diejenigen von ihnen thematisiert werden, die die Systemtheorie in die Musikwissenschaft zu übertragen versuchen, scheint notwendig, am Anfang die Summe der Arbeiten im gewißen Sinn zu differenzieren. Was aber sollte hier als eine Unterscheidung dienen? Wenn man z. B. mit den Kriterien, die von Inhetveen 1997 gebraucht wurde, um die musiksoziologische Arbeiten zu unterscheiden, auswählt, dann könnte man alle die hier vorkommenen Arbeiten nach der disziplinären Bezogenheit ihrer Autoren unterscheiden. So sollten aus der weiteren Diskussion diejenige Arbeiten ausgeschlossen werden, die von Soziologen (z. B. Rotter 1985, Fuchs 1987, Lipp 1992, Fuchs 1992, Rotter 1992, Fuchs 1993a und Fuchs 1996) oder Kommunikationswissenschaftlern (Jacke 1998) stammen. Wenn man aber nach den systemtheoretisch verstandenen kommunikativen Anknüpfungen fragt, sollte man schon diese Arbeiten differenziert sehen: Man könnte sie als Beobachter einerseits, und gleichzeitig als beobachtete Instanzen andererseits sehen. Im ersten Fall sollte man fragen, welche Informationen sie von welchen Mitteilungen unterschieden haben, und im zweiten Fall lautet die Frage, ob es weitere Beobachtungen dieser Beobachtungen gibt. So könnte man kurz sagen, dass die Arbeiten von Peter Fuchs (insbesondere Fuchs 1987, Fuchs 1992 und Fuchs 1996) als die Beobachtungen von, unter anderem, Arbeiten Hanslicks, Riemanns, Dahlhaus', Lehrdahls \& Jackendoffs und Wioras angesehen werden können; auf der anderen Seite stehen die Arbeiten, die als kommunikative Anknüpfungen an die Arbeiten von Fuchs angesehen werden können. Das sind z. B. Rotter 1992, Lipp 1992 und Inhetveen 1997, aber auch Casimir 1991. Besonders Fuchs 1987 wurde von anderen Beobachtern beobachtet und auch oft kritisiert: Der dort entworfenen Konzeption, die Musik mit dem systemtheoretisch verstandenen psychischen System zu relationieren versuchte, wurde z. B. von Rotter vorgeworfen, sie sei einseitig, weil sie Musik als "reinen Zeitzauber" (Rotter 1992: 93) sieht. Rotter selbst findet diese Konzeption unhaltbar, ebenso wie eine strikte Trennung der psychischen von sozialen Systemen (92). Aber die Beobachtungen, könnte man sagen, gehen auch in die Gegenrichtung: So schreibt Fuchs in seinem Aufsatz aus 1992, dass er seine eigenen "Ausgangsprämissen anders als Rotter" (Fuchs 1992: 75) im Buch Rotter 1985 setzt. Während Rotter Musik für ein Kommunikationsmedium im systemtheoretischen Sinn erachtet, schreibt Fuchs: "Sie ist nicht nur nicht Sprache, sie ist auch nicht Kommunikation. Sie muß ihre soziale Bedeutung anders als kommunikativ haben, was nicht ausschließt, dass diese Bedeutung mit Kommunikation zu tun hat." (75) Wenn man aber diese zwei Positionen in Hinsicht auf die schon dargestellten Unterscheidungen zwischen Luhmann und anderen Theoretikern beobachtet, könnte man vermuten, dass die Position der Arbeiten Fuchs 1987 und Fuchs 1992 jener der Luhmanns Sozialen Systeme entspricht, während die Position der Arbeiten Rotter 1985 und Rotter 1992 als bestimmte $A b$ weichung gelten kann: Ihre Kritik an Luhmanns Trennung der psychischen von sozialen Systemen geht konform mit der entsprechenden Kritik in Schmidt 1994. Doch mit der starken Stützung auf die Psychoanalyse, die übrigens sehr heftig kritisiert wurde (z. B. von Lipp 1992 und Inhetveen 1997), unterscheidet sich Rotter von der erwähnten Position Schmidts. 
Beobachtet man wieder mit der Unterscheidung aus Inhetveen 1997, könnte man jetzt die andere Seite wählen, nämlich jene, die die Arbeiten der Musikwissenschaftler bezeichnet. So sollten hier in der weiteren Diskussion als Beispiele der musikwissenschaftlichen Auseinandersetzungen mit der Systemtheorie Luhmanns folgende Arbeiten dienen: Casimir 1991, Gottwald 1991, Mosch 1993, Tadday 1993, Möller 1996, Schläbitz 1997, Schläbitz 1997a, Tadday 1997, Motte-Haber 1998 und Tadday 1998. Wenn zudem in der Diskussion die Frage nach dem Verhältnis zum Radikalen Konstruktivismus aufkommt, könnten als relevant auch Arbeiten wie Großmann 1991, Müllensiefen 1994, Müllensiefen 1998 und die Mehrzahl der Arbeiten aus dem Sammelband Hemker/Müllensiefen 1997 angesehen werden. Die Liste wäre damit wahrscheinlich nicht komplett, aber vielleicht ist in diesem Moment wichtiger, eine Orientierung zu geben, als eine ausführliche Untersuchung durchzuführen. Es scheint auch wichtig anzumerken, dass diese Untersuchung nur mit einer beschränkten Zahl von Kriterien operiert. Deswegen werden jetzt alle anderen Differenzen außer acht gelassen, wie z. B. jene, die die Arbeiten nach Textarten oder Gattungen unterscheiden würden, wie auch jene, die diese Arbeiten nach dem Umfang der Berufung auf die Theorie Luhmanns diskriminieren würden. In diesem zweiten Fall könnte man z. B. Casimir 1991 von Motte-Haber 1998 unterscheiden, da bei Motte-Haber im Vergleich zu Casimir die Theorie Luhmanns eher eine Nebenrolle spielt.

Möchte man aber jetzt versuchen, alle erwähnten Arbeiten systemtheoretisch zu sehen, dann ließe sich die Frage nach der Disziplinbezogenheit auch etwas anders stellen. Wenn man nämlich die Disziplinen als Untersysteme des Wissenschaftssystems sieht, die auf der Möglichkeit beruhen, "die Differenz von System und Umwelt innerhalb des Gesamtsystems zu wiederholen" (Luhmann 1990: 446), dann stellt sich auch die Frage, wie diese Differenzen in diesem konkreten Fall zu begreifen sein sollten. Man könnte sie mit Luhmann als Differenzen zwischen verschiedenen Problemen verstehen: "Die Disziplinbildung orientiert sich an diesem wissenschaftsinternen Problem, sie orientiert sich nicht an unterschiedlichen Gegenstandsfeldern, die vorher schon vorhanden wären und wie Kolonnien nach und nach okkupiert werden. Sie führt deshalb auch nicht zu gegeneinander abgeschlossenen Regionalontologien, sondern bildet ihre Gegenstände nach Maßgabe ihrer Theorien. Das heißt zwar, dass die einzelnen Disziplinen unterschiedliche Phänomenbereiche erfassen, nicht aber, dass die gesellschaftlich konstruierten Dinge wie Länder oder Wolken, Menschen oder Tiere für jeweils nur eine Disziplin konzipiert werden müßten." (451) In diesem Sinn könnte man sagen, dass etwa die Differenz zwischen Fuchs 1987 einerseits und Tadday 1993 andererseits gerade als jene zu begreifen wäre, die zwischen systeminternen Problemen der Soziologie einerseits und der Musikwissenschaft andererseits besteht: Während Fuchs von einem soziologischen Problem ausgeht, und sich mit der Konstruktion der Musik beschäftigt, um dieses Problem zu lösen, versucht Tadday einige systemtheoretischen Ansätze für die befriedigenden Lösungen einiger musikwissenschaftlichen Probleme zu operationalisieren. 
Die Berufung auf die Systemtheorie Luhmanns, um die musikwissenschaftlichen Probleme zu lösen, hat keine lange Tradition. Es scheint aber, dass die Musikwissenschaft für sich selbst relativ schnell Luhmanns Systemtheorie entdeckt hat: Wenn man z. B. entsprechende Beobachtungen innerhalb der Literaturwissenschaft sieht, könnte man sagen, dass sie auch in einer größeren Zahl am Anfang der Neunziger entstanden sind (z. B. Schwanitz 1990, Werber 1992, Schmidt (Hg.) 1993, Plumpe 1993), obwohl es bereits frühere Versuche gab. Als einen der ersten musikwissenschaftlichen Versuche, der am Anfang der Neunziger publiziert wurde, kann man den Entwurf einer musikalischen Systemtheorie von Clytus Gottwald (Gottwald 1991) erwähnen. Diese Arbeit, die die eigene Argumentation - wenn es um Luhmann geht - ausschließlich auf Luhmann 1984 gründet, könnte man als ein Versuch verstehen, die systemtheoretischen Begriffe innerhalb der Musikwissenschaft operationalisierbar zu machen. So unterscheidet er verschiedene Bedeutungen des Systembegriffs (tonales System, Sozialsystem der Musik, Gesellschaftssystem, psychisches System des Komponisten), und problematisiert damit auch die Anwendbarkeit von Luhmanns Sinnbegriff und Kommunikationsbegriff an die schon bestehenden musikwissenschaftlichen Konstrukte, wie z. B. an den "Sprachcharakter der Musik" (Gottwald 1991: 38). Gottwald entscheidet sich zwar für diese Konstrukte, kritisiert aber den Sinn- und Kommunikationsbegriff Luhmanns. Die Argumentation läuft damit teilweise parallel zu jenen theoretischen Richtungen, die die erwähnten Luhmann-Konzepte für unakzeptabel halten. In solchen Fällen ist eine starke Stützung auf die Begriffe und Konzepte von Habermas und besonders von Adorno zu bemerken.

Als eine Beobachtung dieser Arbeit kann man die Ausführungen über Systemtheorie und Komponieren von Ulrich Mosch (Mosch 1993) sehen. Es scheint, dass man dort Gottwald 1991 nicht nur für grundlegend akzeptabel hält, sondern, dass man auch entsprechende Unterscheidungen trifft, um den eigenen Gegenstand zu konstruieren. So wiederholt Mosch die These, dass die "Anwendungen des Systembegriffs auf Musik (...) auf den unterschiedlichsten Ebenen möglich (sind)“ (Mosch 1993: 1), und zwar: "Man kann ein Werk, d. h. seine Bestandsteile in Wechselwirkung, als formales System begreifen. Das hat schon, wenn auch häufig unausgesprochen, Tradition. (...) Man kann auch den Komponisten als Teil eines komplizierten sozialen Systems Musik auffassen, dessen Grenzen sich historisch verlagern, ganz auffälig etwa in unserem Jahrhundert mit der Entstehung der Neuen Musik oder mit der Ausdifferenzierung der zahlreichen sogenannten 'Subkulturen'. Auf einer wiederum anderen Ebene angesiedelt ist das Tonsystem, das, sozial und (wohl auch) in der Natur verankert, werkübergreifend ist und im einzelnen Werk eine je besondere Konkretisierung erfährt." (1) Die Arbeit Gottwald 1991, schreibt Mosch, "der einzige bisher vorliegende Versuch, in Anlehnung an Niklas Luhmann eine 'musikalische Systemtheorie' zu skizzieren, wirft so viele Fragen auf, dass der Platz, um sie in vertretbarer Weise diskutieren zu können, an dieser Stelle bei weitem nicht ausreichen würde. Gegenstand der folgenden Überlegungen soll vielmehr ein Versuch sein, systemtheoretische Vorstellungen kompositorisch fruchtbar zu machen." (1) 
Aus dieser Bemerkung kann man auch sehen, dass Mosch die Arbeit Torsten Casimirs offenbar nicht kennt, ähnlich wie Gottwald, der sie auch nicht erwähnt hat. Bei Casimir 1991 handelt es sich um eine umfassende "systemtheoretische Kritik", wie auch der Untertitel lautet, und zwar eine Kritik an einigen der bestehenden musikwissenschaftlichen Konzepte einerseits und einigermaßen an der Systemtheorie Luhmanns andererseits. Die Arbeit sollte eine "Literaturanalyse" (13) sein: "Ihr Ziel wird die systematische Dokumentation bisheriger Begriffs- und Theoriebildung sein sowie der Versuch, eine Synthese des Materials zu erreichen. Als ihr Orientierungsrahmen dient das Begriffsinventar der Systemtheorie Niklas Luhmanns, insbesondere dessen Kommunikationsbegriff." (13) Aber gleichzeitig wird "in der vorliegenden Untersuchung auch für den Einbezug von Intuition, für die Sensibilität gegenüber 'common sense' und subjektiver Erfahrung des zu erforschenden Gegenstandes plädiert" (18). Deswegen kritisiert Casimir auch die Kommunikationskonzepte, die von Luhmanns Kommunikationsbegriff ausgehen würden: "Alltägliche Musikkommunikation darf dabei nicht als faktisches Ereignis außerhalb von Bewußtseinshorizonten der Kommunikanten angesetzt werden (...), sondern ist zu konzipieren als überhaupt nur durch Erfahrung vorhandene Wirklichkeit. Sie muß aus diesem Grund radikal menschenbezogen untersucht werden. Die Annahme, es gebe Musikkommunikation als spezifisch wissenschaftliche Realität, die autonom von individueller und gesellschaftlicher Wirklichkeit konstituiert sei, ist Fiktion." (60) Eine Folge dieser Kritik ist "eine Modifikation" (216) von einigen Konzepten Luhmanns: "Das Konzept strikter Autopoiese ist aufzuweichen, indem Systeme als teilautonom modeliert werden." (216) An einer anderen Stelle sieht Casimir seine eigenen Bemühungen als eine Kritik der späteren Position Luhmanns, die, nach ihm, mit einer früheren Luhmann-Position ersetzt werden sollte (223). Man könnte aber auch, in Hinsicht auf die schon dargestellten Unterscheidungen, seine Position entsprechend zu jener aus Schmidt 1996 oder auch Schmidt 1991 beobachten, um zu sehen, dass seine Kritik an Luhmann, oder zumindest seine Modifikation von Luhmanns Theorie, mit einigen analogen Argumenten und Lösungen wie entsprechende Theorieansätze Schmidts operiert: Wie Schmidt entscheidet sich Casimir für eine grundsätzlich humanistische Position, er hält auch den Menschen als entscheidende Instanz der Theorie, was dann auch impliziert, dass er auf die radikale Trennung und operationale Geschloßenheit der psychischen und sozialen Systeme verzichten muß. Insofern scheint die Position Casimirs viel näher an Großmann 1991, als man auf dem ersten Blick erwarten könnte, besonders weil diese Arbeit Großmanns, die als eine strenge Anwendung von Schmidt 1991 angesehen werden kann, anscheinend mit der Theorie Luhmanns nichts zu tun hat. Doch man könnte gewiße Übereinstimmung zwischen Casimir 1991 und Großmann 1991 gerade in dem Punkt sehen, in dem beide ihre theoretischen Konstruktionen von einem Menschenbegriff ausgehend konstruieren.

Obwohl Ulrich Tadday in seinem Text von 1997 nicht an Casimir 1991 anknüpft, kommt auch diese Arbeit zu ähnlichen Folgen. Diese "kritische Auseinandersetzung" (Tadday 1997: 14) mit der Systemtheorie Luhmanns beginnt mit der Feststellung, dass Luhmanns Thesen "befremden (müssen), solange Begriffe wie 'Autonomie', 
'Form', 'Umwelt' usw. konventionell und nicht theoriekonform verstanden werden." (14/15) Da "eine spezielle musikwissenschaftliche Auseinandersetzung mit der Luhmannschen Systemtheorie (...) deshalb erst nach einer Übersetzung ihrer generellen Grundlagen möglich (ist)" (15), unternimmt Tadday gerade diesen Schritt. Anschließend kritisiert er: "Luhmanns systemtheoretische Ästhetik reduziert Kunst und Musik auf eine sozialtechnische Dimension, der es selber an Bedeutungstiefe fehlt und die tiefere Bedeutungen von Kunst nicht zuläßt. Gerade am Beispiel der Musik hätte Luhmann (...) erkennen können, wie fragwurdig eine funktionale Definition von Musik ist, die einseitig auf die 'autopoietische Autonomie' des Kunstsystems setzt. Musik 'programmiert' sich nicht ausschließlich selber, sie wird von Menschen komponiert. Für Luhmann existieren diese Menschen nicht mehr als ganze oder Subjekte, sondern nur als strukturelle Koppelung von Teilsystemen, zu denen auch das 'physische System' gehört." (30/31) Wie Taddays Übersetzung, operiert aber auch diese Kritik gerade mit der Unterscheidung, die das musikwissenschaftliche Wissen über Musik vom entsprechenden soziologischen Wissen unterscheidet. Wenn Tadday Luhmanns angebliche Reduktion der Kunst auf ihre sozialtechnische Dimension und seine scharfe Trennung der psychischen von sozialen Systemen kritisiert, argumentiert er gerade aus der Position, aus der er das musikwissenschaftliche Wissen als wahres Wissen über Musik sieht und beherrscht: "Nicht nur im Falle Beethovens erscheint diese Ansicht geradezu als absurd. Wer sich ein wenig in der Musikgeschichte des 19. Jahrhunderts auskennt, weiß, dass die Komponisten von Beethoven bis Mahler ihrer Musik 'poetische Ideen' (...) zugrunde legten, mit ihrer Musik 'Seelenzustände' (...) ausdrücken wollten." (31)

Ein Jahr später hat sich Tadday auch mit der Systemtheorie Luhmanns auseinandergesetzt. Dieses mal beobachtet er Luhmanns Theorie im Vergleich zur Geschichtsphilosophie Hegels, und er findet, dass "sowohl die idealistische als auch systemtheoretische Geschichtsphilosophie (...) die Möglichkeit einer philosophischen Letztbegründbarkeit voraus(setzen). Während die idealistische Philosophie den Satz des letzten Grundes in der Identität von Subjekt und Objekt findet, erhält die Luhmannsche Systemtheorie ihren absoluten Grundsatz durch die Differenz von System und Umwelt." (117) Am Ende zitiert er eine Stelle aus einem Text von Novalis, in dem man alles Suchen nach einem Prinzip mit den Versuchen der Quadratur des Zirkels vergleicht (117).

Die Berufung auf Luhmann in Taddays früherer Veröffentlichung von 1993 scheint auf den ersten Blick anders: Dort ist die Systemtheorie nur eine neben anderen theoretischen Ansätzen, die als theoretischer Rahmen für Taddays Beobachtung dienen. So folgt er z. B. gleichzeitig der Diskurstheorie Foucaults aus seiner Archäologie des Wissens (Tadday 1993: 16), den Kommunikationsbegriff aus Luhmanns Sozialer Systeme (16) und einige Soziologen, die sich stark auf das Habituskonzept Bourdieus stützen (17). Diese Parallelität scheint manchmal so eng, dass man auf einer Seite nebeneinander über den kulturellen Habitus, das soziale System und die diskurziven Formationsregeln (18) lesen kann. Insofern scheint es interessant zu beobachten, wie Tadday weiter mit dieser Kombination von Theorien operiert, besonders wenn man z. B. parallel entsprechende Beobachtungen dieser Theorien in 
anderen Disziplinen sieht (eine systemtheoretische Beobachtung der Literaturtheorien, die sich auf Foucault und Bourdieu stützen, kann man z. B. in Plumpe/Werber 1997 finden). So ist zu bemerken, dass sich Tadday - wenn es um die theoretische Konstruktion der von ihm beobachteten Gesellschaft geht - doch für die Form der gesellschaftlichen Differenzierung entscheidet, die Luhmann für vormoderne Gesellschaften für gültig hält, nämlich für stratifikatorische Differenzierung (Luhmann 1993: 25/26).

Es scheint, dass eine vergleichbare Position mit der aus Tadday 1993 auch in zwei Arbeiten von Norbert Schläbitz gefunden werden kann. Hinsichtlich der schon früher skizzierten Konstruktion der theoretischen Unterscheidungen könnte man den Unterschied zwischen Tadday 1993 und zwei Arbeiten von Schläbitz grob als eine Differenz zwischen den Arten der Modifikation der Theorie Luhmanns sehen: Während bei Tadday einige Konzepte Foucaults und Bourdieus als Faktoren, die eine Modifikation des systemtheoretischen Rahmen verursachen, angesehen werden können, könnte man als bedeutend für die Arbeiten von Schläbitz gerade die Theorie von Siegfried J. Schmidt sehen, z. B. schon dann, wenn er behauptet, dass "menschliche Kommunikation jedweder Art (...) intersubjektiv (ist)" (Schläbitz 1997a: 68).

Am Ende dieses Abschnittes möchte ich noch kurz eine Arbeit von Hartmut Möller erwähnen. Wie bei Tadday 1993 handelt es sich hier um eine Untersuchung, die vom Luhmanns Kommunikationsbegriff ausgeht. Da Möller "gegen unkritische Modelltransfers" (Möller 1996: 97) plädiert, sollte auch die eigene Arbeit einigermaßen kritisch gegenüber der Theorie sein, die als Ausgangspunkt ausgewählt wurde. So kann man am Endes des Aufsatzes folgendes lesen: "Denn welchen Erkenntnisgewinn hat eigentlich die Anwendung des Luhmannschen Kommunikationskonzeptes (...) gebracht, über den Versuch einer Anwendung ungewohnter Beschreibungsweisen hinaus? Dass Begriffe keine feste inhaltliche Qualität haben, sondern ihre je unterschiedliche Bedeutung erst in einer pragmatisch-konkreten Situation erhalten, ist ja als solches auch im Rahmen der Musikwissenschaft keine neue Einsicht. (...) Könnte es sein, dass der Sinn dieses Vorgehens ganz woanders zu suchen ist: Nämlich in dem Angebot zu einer kritischen Distanzierung von unserem gewohnten Umgang mit musikgeschichtlichen Texten, indem einiges von dem, was uns in unseren Geschichtskonstruktionen selbstverständlich ist, als Besonderheit unserer Beobachtungsweise sichtbar gemacht werden kann? Niklas Luhmann spricht öfters von einer 'Beobachtung zweiter Ordnung' (...).dass wir grundsätzlich nie mit der Wirklichkeit an sich umgehen, sondern stets mit unseren Erfahrungswirklichkeiten, ist seit Vico und Kant bekannt - die Frage ist nur, welche Konsequenz wir aus dieser Einsicht ziehen (...). Folgen wir, wie neuerdings USamerikanische 'New Musicology' mit vollen Segeln Derridas philosophischer Aufwertung der sprachlichen Fiktion zum letzten 'Wahrheitsmedium' in einer dekonstruktiv fiktionalisierten Welt? (...) Oder ist stattdessen in unseren Forschungsinteressen bisweilen die Anwendung und Mischung von bestimmten theoretischen Ansätzen und Systenkonzepten so sehr in den Vordergrund gerückt, dass darüber die Suche nach mittelbaren Erkenntnisgewinn in der Beschreibung, Modellierung, Erk- 
lärung und Deutung bestimmter Objektbereiche mehr und mehr in den Hintergrund tritt, vergessen und gar negiert wird? Oder versuchen wir auch weiterhin, über eine (...) zeitbegrenzte Konzentration auf Probleme von Textwelten, Diskursen, narrativen Strukturen, Deutungsschemata, Systemtheorien usw. hinaus jene Verantwortung nicht aus dem Blick zu verlieren, in die auch die Wissenschaften eingebunden sind?" (98/100) In Hinsicht auf diese Fragen, aus denen zuerst zu entnehmen ist, dass die Systemtheorie Luhmanns von Möller als eine Abschafftung des "ontologischen Gegensatzes" (97) zwischen Fiktion und Wirklichkeit verstanden wurde, wurde auch eine Antwort gegeben: "(Musik-) Geschichte bezieht sich eben erfahrungsgemäß nicht nur auf Sprachspiele in einer fiktionalisierten Scheinwelt, sondern ist auch eine wirkmächtige, orientierende Lebensmacht." (100)

\section{Diskussion}

Ich möchte am Ende noch einmal an die Thesen von Fohrmann und Krims erinnern, um sich zu fragen, ob es möglich ist, über ihre Bestätigung durch die dargestellten Beispiele zu sprechen. Könnte man also sagen, dass die musikwissenschaftlichen Theorie-Ansätze, die hier beobachtet wurden, keineswegs zufällige oder willkürliche Form hatten, sondern, dass sie notwendigerweise, so Jürgen Fohrmann, auf der Basis der schon bestehenden disziplinären Matrix operierten? Was sollte aber in der Musikwissenschaft als diese Matrix fungieren? Krims hat sie in der Idee der musikalischen Autonomie gefunden; die musikwissenschaftlichen Beobachtungen, so Krims, haben die Dekonstruktion Derridas so reduziert, dass sie genau der Fortsetzung dieser Idee dienen könnten. Läßt sich also sagen, dass die Musikwissenschaft tatsächlich von der "ideology of autonomous art" (Wolff 1987) bezaubert ist, und sollte man, wie das z. B. bei Krims der Fall ist, die Musikwissenschaft dafür kritisieren? Was könnte ein (so würde das Möller formulieren) Erkenntnisgewinn sein, wenn man Musikwissenschaft von dieser Ideologie zu befreien versucht? Sieht man tatsächlich besser, wenn man, statt nach dem "self-relatedness of musical elements" (Krims 1998: 320), nach den "sociopolitical dimensions of musical life" (320) sucht? Bevor man aber auf diese Fragen zu antworten versucht, sollte man vielleicht nicht vergessen, dass die Beobachtungen von Krims nur als sehr partiell angesehen werden können: Er hat nur eine kleine Auswahl aus der musikwissenschaftlichen Produktion beobachtet, und zwar so, dass ausgewählte Arbeiten allein hinsichtlich einer Differenz beobachtet wurden, nämlich jener: Textuell/nicht-textuell. Auch sollte vielleicht nicht vergessen werden, dass sowohl diese Arbeiten, als auch die Beobachtungen von Krims, innerhalb der US-amerikanischen Musikwissenschaft funktionieren. Da man ähnliche Beschränkungen auch bei den Beobachtungen, die hier unternommen wurden, finden kann, sollte man doch nicht allzuschnell die Resultate generalisieren.

Man kann aber noch eine Frage an Krims stellen: Wie kann er überhaupt die musikwissenschaftlichen Beobachtungen der Dekonstruktion so sehen, wie er sie gesehen hat, nämlich als eine gewiße Reduktion derselben Theorie? Wie kann er sich außerhalb der Musikwissenschaft positionieren, um die wahre Dekonstruktion zu sehen, und sich gleichzeitig innerhalb der Musikwissenschaft befinden, um einen 
Beitrag zur Musikwissenschaft zu leisten? Wie kann man auf diese Weise die musikwissenschaftlichen Beobachtungen der soziologischen Systemtheorie beobachten, die hier unternommen wurden? Man könnte mit Luhmann antworten: Weil es sich um einen re-entry handelt. Diesen Begriff hat Luhmann von Logiker George Spencer Brown übernommen, um einen theoretischen Umgang mit den Paradoxien zu ermöglichen. Wie konkret der re-entry läuft, hat er wie folgt beschrieben: "Eine Unterscheidung markiert einen Bereich und wird dann in das durch sie Unterschiedene wiedereingeführt. Sie kommt dann doppelt vor: Als Ausgangsunterscheidung und als Unterscheidung in dem durch sie Unterschiedenen. Sie ist dieselbe und nicht dieselbe. Sie ist dieselbe, weil der Witz des re-entry gerade darin besteht, dieselbe Unterscheidung rekursiv auf sich selbst anzuwenden; sie ist eine andere, weil sie in einen anderen, in einen bereits unterschiedenen Bereich eingesetzt wird." (Luhmann 1990: 379/380) Das heißt konkret, dass die Musikwissenschaft, folgt man Luhmann, die Unterscheidung zwischen sich selbst und einer anderen Disziplin wieder in sich selbst einführen kann. Diese Operation innerhalb der Systemtheorie Luhmanns setzt aber gleichzeitig voraus, dass die Musikwissenschaft mit dieser Operation sich selbst wie ein autopoietisches System weiter reproduziert. Damit scheint es auch wichtig zu betonen, dass es sich beim re-entry der Differenz soziologische Theorie/musikwissenschaftliche Theorie keineswegs um eine Beobachtung der Musikwissenschaft handelt, die von der Seite der soziologischen Theorie ausgehen würde: Auch die soziologische Theorie, wie sie hier dargestellt wurde, ist ein musikwissenschaftliches Konstrukt.

Nach Luhmann wäre es jetzt möglich, diese musikwissenschaftliche Beobachtung der soziologischen Theorie mit einer neuen Unterscheidung weiter zu beobachten, um zu sehen, wie sie überhaupt diese soziologische Theorie reduziert hat. Und auch diese Beobachtung, als eine neue Beobachtung zweiter Ordnung, würde mit einer Unterscheidung operieren, die sie selbst nicht beobachten kann, die aber als ein blinder Fleck von einem neuen Beobachter beobachtet werden kann. Die Systemtheorie Luhmanns konstruiert in dieser Weise die Wissenschaft als ein autopoietisches System, das sich selbst immer weiter reproduziert, indem es den blinden Fleck einer anderen wissenschaftlichen Beobachtung beobachtet. Diese Konzeption der rekursiven Beobachtungen rechnet mit der Vorstellung, nach der das Wissen immer von einen Beobachter mit einer Unterscheidung konstruiert wird und insofern keinen direkten Zugang zur Wirklichkeit hat, weil diese Beobachtung immer nur das sieht, was sie mit dieser Unterscheidung sehen kann. Deswegen hat Luhmann immer wieder betont, dass Beobachtung keineswegs mit Ideologiekritik verwechselt werden darf, da die Kritiker der Ideologie von der Voraussetzung ausgehen, dass sie selbst den wahren Zugang zur Wirklichkeit haben, und insofern alle andere Zugänge als falsches Bewußtsein kritisieren können, während das Beobachtungskonzept, nach Luhmann, immer mit einem blinden Fleck der Beobachtung rechnet, also auch mit dem blinden Fleck der eigenen Beobachtung. Die Beobachtung sollte aber auch nicht mit blankem Pluralismus verwechselt werden: "Auf der Suche nach einer Alternative zu einer bloßen Historisierung und Standpunktrelativierung allen Wissens (...), wählen wir deshalb einen anderen Weg, der 
uns zugleich in Distanz bringt zur üblichen Akzeptanz von 'Pluralismus'. Unser Ausgangspunkt liegt bei einem extrem formalen Begriff des Beobachtens, definiert als Operation des Unterscheidens und Bezeichnens." (Luhmann 1990: 73) So kann die Beobachtung zuerst die Unterscheidung sehen, mit der eine andere Beobachtung operiert, und dann sieht sie die Seite der Unterscheidung, die nicht ausgewählt wurde. In diesem Sinn kann sie, nach Luhmann, die Kontingenz des Beobachteten sehen, ohne dass sie gleichzeitig eine bloße Historisierung unternimmt.

Luhmann selbst hat hinsichtlich des Beobachtungskonzeptes mehrmals behauptet, dass die Ideologiekritik keineswegs einen Blick aus einer privilegierten Position zuläßt, als die aus der Ideologie selbst (Luhmann 1989: 11). Wenn alle Beobachtungen mit einen blinden Fleck operieren, dann sei es nicht möglich zu sagen, welche Beobachtung mehr als die andere sieht. Daniel Barben sieht gerade hier eine Theorieentscheidung Luhmanns, die nicht unbedingt so sein muß: "Luhmann (verbucht) die Einsicht in die notwendige Beschränktheit jeder Sicht in der Weise für seinen Theorieansatz, dass deren eigene Beschränktheit legitimiert, ja mitunter sogar zu einer Auszeichnung verkehrt wird. Denn wenn man als 'Kybernetiker zweiter Ordnung' zwar weiß, dass man nicht weiß, was man nicht weiß, weil man es nicht weiß, heißt das nicht zugleich, dass man nicht sehen kann, was man nicht sehen kann, weil man so sieht: es gibt also verschiedene Arten von blinden Flecken." (Barben 1997: 240) Es scheint, dass Luhmann selbst dieses Problem thematisiert hat, wenn er in einem seiner späteren Texten geschrieben hat, dass es "die Aufgabe der Wissenssoziologie (...) sein könnte, die Bedingungen zu erforschen, unter denen bestimmte Unterscheidungen mehr einleuchten als andere." (Luhmann 1999: 176)

Die hier unternommene Beobachtung der Musikwissenschaft kann auch als ein Konstrukt in Hinsicht auf die theoretischen Vorentscheidungen angesehen werden. Folgt man Luhmann, könnte man sagen, dass diese Beobachtung nur das sehen kann, was sie mit ihren theoretischen Instrumenten als eigenen Gegenstand konstruiert. Wären andere theoretische Vorentscheidungen ausgewählt, wäre auch der Gegenstand der Untersuchung anders: Wäre z. B. die Wissenssoziologie Bourdieus - wie sie in Bourdieu 1988, Bourdieu 1994 und Bourdieu 1997 entworfen wurde als theoretischer Rahmen ausgewählt, wäre der Gegenstand der Untersuchung keine systemtheoretisch verstandene Beobachtung, sondern das wissenschaftliche Feld, das "all jene Akteure und Institutionen umfaßt, die (...) Wissenschaft erzeugen und verbreiten." (Bourdieu 1997: 18) Aber dann wäre auch der blinde Fleck anders. Es ist nur zu hoffen, dass die hier unternommene Darstellung, zusammen mit ihrem blinden Fleck, genug anregend wirken wird, um die weiteren kommunikativen Anknüpfungen zu erfassen.

\section{BIBLIOGRAPHIE}

ADORNO, Theodor W. 1962. "Vermittlung", in: ders. Einleitung in die Musiksoziologie: Zwölf theoretische Vorlesungen. Frankfurt/M.

BAECKER, Dirk 1999. "Wenn etwas der Fall ist, steckt auch etwas dahinter", in: Stichweh, Rudolf (Hg.) Niklas Luhmann - Wirkungen eines Theoretikers: Gedenkcolloquium der Universität Bielefeld am 8. Dezember 1998. Bielefeld.

BARBEN, Daniel 1996. Theorietechnik und Politik bei Niklas Luhmann: Grenzen einer universalen Theorie der modernen Gesellschaft. Opladen. 


\section{MUZIKOLOŠKI ZBORNIK • MUSICOLOGICAL ANNUAL XXXV}

BARSCH, Achim/RUSCH, Gebhard/VIEHOFF, Reinhold (Hg.) 1994. Empirische Literaturwissenschaft in der Diskussion. Frankfurt/M.

BERG, Henk de/PRANGEL, Matthias (Hg.) 1995. Differenzen: Systemtheorie zwischen Dekonstruktion und Konstruktivismus. Tübingen.

BEYER, Martin 1996. Kontakt über Theorie: Studien zur theologischen Rezeption der Systemtheorie Niklas Luhmanns. Diss. Univ. Leipzig.

BLOM, Tannelie/NIJHUIS, Ton 1995. "Sinn und Kunst: Die Umarmung Niklas Luhmanns durch die Literaturtheorie und Kunstgeschichte", in: Berg/Prangel (Hg.) 1995.

BOURDIEU, Pierre 1988. Homo academicus (1984). Frankfurt/M.

BOURDIEU, Pierre 1994. Die feinen Unterschiede: Kritik der gesellschaftlichen Urteilskraft (1979). Frankfurt/M.

BOURDIEU, Pierre 1997. Vom Gebrauch der Wissenschaft: Für eine klinische Soziologie des wissenschaftlichen Feldes. Konstanz.

BRAUN, Christoph 1992. Max Webers "Musiksoziologie. Laaber.

CASIMIR, Torsten 1991. Musikkomunikation und ihre Wirkungen: Eine systemtheoretische Kritik. Wiesbaden.

DALLMANN, Hans-Ulrich 1994. Die Systemtheorie Niklas Luhmanns und ihre theologische Rezeption. Stuttgart/Berlin/Köln.

EDSTRÖM, Olle 1997. "Fr-a-g-me-n-ts: A discussion on the position of critical ethnomusicology in contemporary musicology“, Svensk tidskrift för musikforskning 79.

ESPOSITO, Elena 1998. "Operation/Beobachtung", in: Baraldi, Claudio/Corsi, Giancarlo/dies. Glossar zu Niklas Luhmanns Theorie sozialer Systeme. Frankfurt/M.

FEDER, Georg 1980. „Empirisch-experimentelle Methoden in der Musikforschung: Kritische Bemerkungen zur Kompetenz und Eigenständigkeit der Systematischen Musikwissenschaft und zur Relevanz einiger ihrer Ergebnisse“, Die Musikforschung 33.

FOHRMANN, Jürgen 1997. "Einleitung،, in: ders./Müller (Hg.) 1997.

FOHRMANN, Jürgen/MÜLLER, Harro (Hg.) 1997. Systemtheorie der Literatur. München.

FUCHS, Peter 1987. "Vom Zeitzauber der Musik: Eine Diskussionsanregung “, in: Baecker, Dirk/Markowicz, Jürgen/Stichweh, Rudolf/Tyrell, Hartmann/Willke, Helmut (Hg.) Theorie als Passion: Niklas Luhmann zum 60. Geburtstag. Frankfurt/M.

FUCHS, Peter 1992. "Die soziale Funktion der Musik", in: Lipp (Hg.) 1992.

FUCHS, Peter 1993. Moderne Kommunikation: Zur Theorie des operativen Displacements. Frankfurt/M. FUCHS, Peter 1993a. Niklas Luhmann - beobachtet. Opladen.

FUCHS, Peter 1996. "Musik und Systemtheorie - Ein Problemaufriß", in: Polaschegg, Nina/Hager, Uwe/Richtsteig, Tobias (Hg.) Diskurse zur gegenwärtigen Musikkultur (= Forum Musik Wissenschaft, Bd. 3). Regensburg.

GOTTWALD, Clytus 1991. "Erkenntnisgenuß: Entwurf einer musikalischen Systemtheorie«. MusikTexte 38. GROSSMANN, Rolf 1991. Musik als "Kommunikation": Zur Theorie musikalischer Kommunikationshandlungen. Braunschweig.

GUMBRECHT, Hans-Ulrich 1988. "Who is Afraid of Deconstruction? «, in: Fohrmann, Jürgen/Müller, Harro (Hg.) Diskurstheorien und Literaturwissenschaft. Frankfurt/M.

HABERMAS, Jürgen 1971. "Theorie der Gesellschaft oder Sozialtechnologie?: Eine Auseinandersetzung mit Niklas Luhmann", in: ders./Luhmann 1971.

HABERMAS, Jürgen 1981. Theorie des kommunikativen Handelns, 2 Bde. Frankfurt/M.

HABERMAS, Jürgen/LUHMANN, Niklas 1971. Theorie der Gesellschaft oder Sozialtechnologie-Was leistet die Systemforschung?. Frankfurt/M.

HELLE, Horst Jürgen 1992. "Musik als Thema bei Georg Simmel und Max Weber", in: Lipp (Hg.) 1992.

HEMKER, Thomas/MÜLLENSIEFEN, Daniel (Hg.) 1997. Medien - Musik-Mensch: Neue Medien und Musikwissenschaft. Hamburg.

INHETVEEN, Katharina 1997. Musiksoziologie in der Bundesrepublik Deutschland: Eine kritische Bestandsaufnahme. Opladen. 


\section{MUZIKOLOŠKI ZBORNIK • MUSICOLOGICAL ANNUAL XXXV}

JACKE, Christoph 1998. "Millionenschwere Medienverweigerer: Die US-Rockband Nirvana“, in: Rösing, Helmut/Phleps, Thomas (Hg.) Neues zum Umgang mit Rock- und Popmusik (= Beiträge zur Popularmusikforschung 23). Karben.

KADEN, Christian 1997. "Musiksoziologie", MGG, Sachteil 6.

KINGSBURY, Henry 1988. Music, Talent, and Performance: A Conservatory Cultural System. Philadelphia.

KINGSBURY, Henry 1991. "Sociological Factors in Musicological Poetics", Ethnomusicology 35.

KOLLERITSCH, Otto (Hg.) 1998. Abschied in die Gegenwart: Teleologie und Zuständlichkeit in der Musik (= Studien zur Wertungsforschung, Bd. 35). Wien/Graz.

KRIMS, Adam 1998. "Disciplining Deconstruction (For Music Analysis)", 19th Century Music 21.

KRIMS, Adam 1998a. "Introduction: Postmodern Musical Poetics and the Problem of 'Close Reading', in: ders. (Hg.) 1998. Music/Ideology: Resisting the Aesthetic. Amsterdam.

LIPP, Wolfgang 1992. "Gesellschaft und Musik: Zur Einführung ", in: ders. (Hg.) 1992.

LIPP, Wolfgang (Hg.) 1992. Gesellschaft und Musik: Wege zur Musiksoziologie (= Sociologia internationalis, Beiheft 1). Berlin.

LUHMANN, Niklas 1971. "Systemtheoretische Argumentationen: Eine Entgegnung auf Jürgen Habermas", in: Habermas/ders. 1971.

LUHMANN, Niklas 1981. "Unverständliche Wissenschaft: Probleme einer theorieeigenen Sprache“, in: ders. Soziologische Aufklärung 3: Soziales System, Gesellschaft, Organisation. Opladen.

LUHMANN, Niklas 1984. Soziale Systeme: Grundriß einer allgemeinen Theorie. Frankfurt/M.

LUHMANN, Niklas 1989. "Reden und Schweigen", in: ders./Fuchs, Peter Reden und Schweigen. Frankfurt/M.

LUHMANN, Niklas 1990. Die Wissenschaft der Gesellschaft. Frankfurt/M.

LUHMANN, Niklas 1990a. Soziologische Aufklärung 5: Konstruktivistische Perspektiven. Opladen.

LUHMANN, Niklas 1993. "Gesellschaftliche Struktur und semantische Tradition" (1980), in: ders. Gesellschaftstruktur und Semantik: Studien zur Wissenssoziologie der modernen Gesellschaft, Bd. 1. Frankfurt/M.

LUHMANN, Niklas 1994. "Die Tücke des Subjekts und die Frage nach den Menschen", in: Fuchs, Peter/Göbel, Andreas (Hg.) Der Mensch - das Medium der Gesellschaft?. Frankfurt/M. (Nachdruck in: Luhmann 1996)

LUHMANN, Niklas 1995. Die Kunst der Gesellschaft. Frankfurt/M.

LUHMANN, Niklas 1996. Soziologische Aufklärung 6: Die Soziologie und der Mensch. Opladen.

LUHMANN, Niklas 1999. "Die Soziologie des Wissens: Probleme ihrer theoretischen Konstruktion", in: ders. Gesellschaftstruktur und Semantik: Studien zur Wissenssoziologie der modernen Gesellschaft, Bd. 4. Frankfurt/M.

MANNHEIM, Karl 1965. "Wissenssoziologie" (1931), in: ders. Ideologie und Utopie. Frankfurt/M.

MATURANA, Humberto 1996. "Biologie der Sozialität" (1985), in: Schmidt (Hg.) 1996.

MÖLLER, Hartmut 1996. "Bernhard von Clairvaux und die Kommunikation über musikalische Umbrüche seiner Zeit: Systemtheorie und Musikgeschichtsforschung “, in: Heister, Hanns-Werner (Hg.) Musik/Revolution: Festschrift für Georg Knepler zum 90. Geburtstag, Bd. 1. Hamburg.

MOSCH, Ulrich 1993. "Systemtheorie und Komponieren: Anmerkungen zum Karlheinz Essl's kompositorischem AnsatZ", http://www.essl.at/bibliogr/mosch.html

MOTTE-HABER, Helga de la 1998. "Freiräume in der Gegenwart - Horror vacui für die Zukunft?: Musik am Ende des Jahrtausends", in: Kolleritsch (Hg.) 1998.

MÜLLENSIEFEN, Daniel 1994. "Der Radikale Konstruktivismus als forschungsmodullierendes Paradigma in der Musikwissenschaft", Manuskript.

MÜLLENSIEFEN, Daniel 1998. "Radikaler Konstruktivismus und Musikwissenschaft: Ideen und Perspektiven", Manuskript.

NASSEHI, Armin 1992. "Wie wirklich sind die Systeme?: Zum ontologischen und epistemologischen Status von Luhmanns Theorie sozialer Systeme", in: Krawiez, Werner/Welker, Michael (Hg.) Kritik der Theorie sozialer Systeme: Auseinandersetzung mit Luhmanns Hauptwerk. Frankfurt/M.

NORRIS, Christopher 1988. Deconstruction and the Interests of Theory. London.

PLUMPE, Gerhard 1993. Ästhetische Kommunikation der Moderne, 2 Bde. Opladen. 


\section{MUZIKOLOŠKI ZBORNIK • MUSICOLOGICAL ANNUAL XXXV}

PLUMPE, Gerhard/WERBER, Niels 1997. "Systemtheorie in der Literaturwissenschaft oder 'Herr Meier wird Schriftsteller' “, in: Fohrmann/Müller (Hg.) 1997

REINECKE, Hans-Peter 1993. "Musikwissenschaft, Systematische Musikwissenschaft, Musikgeschichte Versuch einer Bilanz", Systematische Musikwissenschaft 1.

ROTTER, Frank 1985. Musik als Kommunikationsmedium: Soziologische Medientheorien und Musiksoziologie. Berlin.

ROTTER, Frank 1992. "Kultursoziologische Perspektiven musikalischen Ausdrucks", in: Lipp (Hg.) 1992.

SCHLÄBITZ, Norbert 1997. "Medien - Musik - Mensch", in: Hemker/Mullensiefen (Hg.) 1997.

SCHLÄBITZ, Norbert 1997a. Der diskrete Charme der Neuen Medien: Digitale Musik im medientheoretischen Kontext und deren musikpädagogische Wertung. Augsburg.

SCHMIDT, Siegfried J. 1991. Grundriß der empirischen Literaturwissenschaft (1980). Frankfurt/M.

SCHMIDT, Siegfried J. 1994. Kognitive Autonomie und soziale Orientierung: Konstruktivistische Bemerkungen zum Zusammenhang von Kognition, Kommunikation, Medien und Kultur. Frankfurt/M.

SCHMIDT, Siegfried J. 1995. "Konstruktivismus, Systemtheorie und Empirische Literaturwissenschaft: Anmerkungen zu einer laufenden Debatte", in: Berg/Prangel (Hg.) 1995.

SCHMIDT, Siegfried J. 1996. "Der Radikale Konstruktivismus: Ein neues Paradigma im interdisziplinären Diskurs" (1987), in: ders. (Hg.) 1996.

SCHMIDT, Siegfried J. 1998. Die Zähmung des Blicks: Konstruktivismus - Empirie- Wissenschaft. Frankfurt/M.

SCHMIDT, Siegfried J. (Hg.) 1993. Literaturwissenschaft und Systemtheorie: Positionen, Kontroversen, Perspektiven. Opladen.

SCHMIDT, Siegfried J. (Hg.) 1996. Der Diskurs des Radikalen Konstruktivismus (1987). Frankfurt/M.

SCHNEIDER, Wolfgang Ludwig 1998. "Peter Fuchs - beobachtet", in: Bardmann, Theodor M. (Hg.) Zirkuläre Positionen 2: Die Konstruktion der Medien. Opladen.

SCHWANITZ, Dietrich 1990. Systemtheorie und Literatur: Ein neues Paradigma. Opladen.

SILBERMANN, Alphons 1958. "Die Stellung der Musiksoziologie innerhalb der Soziologie und der Musikwissenschaft", Kölner Zeitschrift für Soziologie und Sozialpsychologie 10.

SILBERMANN, Alphons 1963. "Die Pole der Musiksoziologie“, Kölner Zeitschrift für Soziologie und Sozialpsychologie 15.

SIMMEL, Georg 1975. "Psychologische und ethnologische Studien über Musik" (1882), in: Kneif, Tibor (Hg.) Texte zur Musiksoziologie. Köln.

SIMMEL, Georg 1989. „Über sociale Differenzierung: Sociologische und psychologische Untersuchungen" (1890), in: ders. Gesamtausgabe, Bd. 2. Frankfurt/M.

TADDAY, Ulrich 1993. Die Anfänge des Musikfeuilletons: Der kommunikative Gebrauchswert musikalischer Bildung in Deutschland um 1800. Stuttgart/Weimar.

TADDAY, Ulrich 1997. "Systemtheorie und Musik: Luhmanns Variante der Autonomieästhetik", Musik und Ästhetik 1 .

TADDAY, Ulrich 1998. "Der schwere und der leichte Abschied: Vergangenheit und Zukunft in der Gegenwart von Luhmanns Systemtheorie ", in: Kolleritsch (Hg.) 1998.

WEBER, Max 1921. Die rationalen und soziologischen Grundlagen der Musik. München.

WERBER, Niels 1992. Literatur als System: Zur Ausdifferenzierung literarischer Kommunikation. Opladen.

WOLFF, Janet 1987. "Foreword: The Ideology of autonomous Art", in: Leppert, Richard/McClary Susan (Hg.) Music and Society: The Politics of Composition, Performance and Reception. Cambridge. 
MUZIKOLOŠKI ZBORNIK • MUSICOLOGICAL ANNUAL XXXV

\section{Primeri oblikovanja teorije $v$ muzikologiji}

\section{Povzetek}

Ena od pobud za nastanek pričujočega prispevka je problematika znanstvene interdisciplinarnosti oziroma pretoka teorij iz ene discipline $v$ drugo, kakor jo je zaslediti $v$ objavah sociologa Niklasa Luhmanna. Ce nadalje sledimo pripombi muzikologa H.-P. Reineckeja, da je treba muzikologijo podvreči "opazovanju drugega reda", orišemo nalogo lastnega dela: opazovati, kako muzikologija opazuje konkretno teorijo, na tem mestu Luhmannovo sistemsko teorijo. Tudi samo opazovanje opazovanja pri tem izhaja iz teoretičnega inštrumentarija Luhmannove teorije oziroma iz orisa medsebojnih razmerij med sistemsko teorijo in nekaterimi drugimi teorijami. Opazovanje muzikoloških del (C. Gottwalda, U. Moscha, T. Casimira, U. Taddaya, N. Schläbitza in H. Möllera) razkriva, da le-ta bolj ali manj modificirajo Luhmannovo sistemsko teorijo, večidel tako, da npr. ne sprejmejo njegove teze o ločenosti psihičnih in socialnih sistemov. V sklepni diskusiji je predmet razmisleka teza književnega teoretika J. Fohrmanna, po kateri omogočajo opazovanje druge discipline le obstoječe disciplinarne matrice. Ob koncu skušam s pomočjo sistemsko-teoretičnega pojma "reentry" ugotoviti položaj lastnega opazovanja. 